\title{
Editorial
}

\section{Heart rate variability and cardiac failure}

Time and frequency domain analysis of heart rate variability (HRV) has provided adequate information for risk stratification and evaluation of autonomic tone after myocardial infarction. ${ }^{1}$ It is therefore not surprising that this non-invasive investigation has been used in patients with cardiac failure - that is, in clinical conditions often characterised by signs of neurohumoral sympathetic activation.

This article reviews some of the most interesting and recent results obtained with the analysis of HRV in patients with cardiac failure, focusing on two principal aspects: the detection of an abnormal autonomic modulation of sinus node, and the prognostic value of reduced HRV.

\section{HRV and neurohumoral activation in cardiac failure}

In patients with cardiac failure, when either short term or 24 hour recordings were analysed, reduced HRV was consistently observed and interpreted as a result of predominantly sympathetic and reduced vagal modulation of sinus node. ${ }^{1}$ It was also reported that the extent of reduction of time domain measures of HRV could be correlated with indices of severity of the disease, whereas the relation between the results of spectral analysis of HRV and the different degrees of severity of cardiac failure appeared more complex and of not unique interpretation. Compared with normal subjects, ${ }^{12}$ patients with mild cardiac failure presented a significant increase in low frequency (LF) and a reduction in high frequency (HF) power, whereas RR variance was only slightly decreased-that is, a spectral pattern consistent with that observed in most clinical conditions characterised by enhanced sympathetic and reduced vagal modulation of sinus node. ${ }^{3}$ On the contrary, the power spectrum of patients in the most advanced phases of the disease ${ }^{1-4}$ showed a progressive reduction of the energy in the LF and HF range, with most of the residual power distributed within the very low frequency (VLF) band. Thus, at variance with New York Heart Association (NYHA) functional class I-II patients, the spectral profile of NYHA class IV patients did not reflect the clinical picture of neurohumoral sympathetic activation, as it was characterised by a pronounced reduction in total power rather than by a predominance of the LF component. A possible but unproved explanation for this finding is a change in the responsiveness of pacemaker cells to neural inputs as a result of a persistent neurohumoral sympathetic activation that is not opposed, at the sinus node level, by modulatory vagal activity. ${ }^{5}$ This interpretation was originally proposed to explain the presence of similar results in postmyocardial infarction patients with depressed left ventricular function. ${ }^{16}$ An alternative hypothesis supported by recent findings ${ }^{7}$ is that an abnormal and irregular respiratory pattern might exert an effect on HRV at different frequency ranges. It has been reported ${ }^{7}$ that a discrete VLF component, accounting for almost $70 \%$ of total power, could be ascribed to an abnormal respiratory pattern that can be recognised in most patients with an advanced degree of cardiac failure. In these subjects, respiration may influence directly, as well as through indirect mechanical and haemodynamic effects, the beat to beat variability of heart period thus making more complex the interpretation of the detected LF and HF components. For these reasons, spectral analysis of short term recordings seems of limited value to provide information not only on autonomic modulation of sinus node but also on a prognostic factor such as the extent of systemic neurohumoral activation.

It is a common observation that a drastic reduction in time and frequency domain measures of HRV can be observed when 24 hour Holter recordings of patients with cardiac failure are analysed, and that pharmacological interventions associated with an improvement of clinical conditions are accompanied by a partial and incomplete normalisation of HRV. ${ }^{1}$ The amount of observed changes, however, is so variable that its clinical value is far from established.

To overcome some of these limitations, efforts have been addressed to the development and application of new methods aimed to evaluate the non-linear characteristics of HRV signal, in particular its complexity. ${ }^{1}$ This approach, which has in the Poincare plot its most simple geometrical application, appears too complex and not sufficiently standardised to be used in the clinical setting. In addition, at present it does not provide information on cardiac neural control over and above that derived by time or frequency domain analysis of HRV.

\section{HRV and risk stratification}

\section{BACKGROUND}

The possibility of using HRV as a prognostic indicator in patients with cardiac failure, as reported for postmyocardial infarction patients, ${ }^{1}$ stems directly from the compelling evidence that has been accumulated over the past few years about the importance of the derangement of autonomic nervous control of the cardiovascular system as one of the mechanisms involved in the occurrence of cardiacparticularly sudden-death. Experimental and clinical studies have reported that both increased sympathetic and decreased parasympathetic tone may interact with the electrophysiological mechanisms underlying arrhythmogenesis. Nevertheless, as other factors such as abnormal ventricular wall stretch, electrolyte disorders, and proarrhythmic effects of drugs may play an important role in the genesis of life threatening ventricular arrhythmias and sudden death in cardiac failure patients, the role of autonomic nervous system dysfunction in the pathogenesis of fatal events is less clear than in patients with coronary artery disease. However, the fact that reduced HRV in postmyocardial infarction patients appears to predict not only sudden death but also total cardiac mortality, ${ }^{1}$ suggests that, at clinical level, sympathetic hyperactivity in addition to increased electrical instability may combine and have multiple deleterious effects on all abnormal substrates leading to cardiac events.

\section{CLINICAL DATA}

The most relevant studies that have addressed the role of HRV in risk stratification of patients with cardiac failure will be considered. They differ profoundly in terms of number of patients enrolled, selection of parameters, and statistical methods. As a result, it is currently not known whether measures of HRV are useful and independent predictors of outcome in patients with cardiac failure. 
In 1992, Binder et al reported for the first time that reduced HRV was associated with a 20 -fold increased risk of death. ${ }^{8}$ In the following years this observation was confirmed by other reports. ${ }^{9-13}$ However, the populations included in these studies were often very small with a limited number of events, and correct multivariate regression analysis was not always applied.

Among the HRV parameters selected to stratify cardiac failure patients, an altered non-parametric measure such as the Poincare plot was also proposed..$^{12}$ The main limitation of this index is the subjective criteria of the investigator in the classification of the plots as normal or abnormal, particularly in the absence of any quantification of the data.

Recently, two other studies ${ }^{14}{ }^{15}$ that focused on the value of HRV in the prediction of cardiac death, found that a decreased 24 hour standard deviation of normal RR intervals (SDNN) (nearly below $100 \mathrm{~ms}$ ) was related to an increased risk of cardiac death. However, only in the study by Ponikowski et al was this index independent of other clinical factors such as peak oxygen consumption, NYHA class, left ventricular ejection fraction, and occurrence of ventricular tachycardia during Holter recording. ${ }^{15}$ The main limitation of these studies, besides the small number of events, is the choice of the cut off value defining normal and reduced HRV. These two studies ${ }^{14}{ }^{15}$ used a cut off of $100 \mathrm{~ms}$, which included about $40 \%$ of the enrolled population and was at variance with the indication of the task force on $\mathrm{HRV},{ }^{1}$ which suggested lower values for patients with a depressed HRV.

A value of SDNN $<50 \mathrm{~ms}$ was reported to distinguish a subgroup of severe congestive heart failure patients with a shorter event free survival time, even when conventional measures such as ejection fraction, cardiothoracic ratio, and number of extrasystoles did not differ between patients with and without cardiac events. ${ }^{16}$ Unfortunately the size of the study was small.

As to the possible predictive value of the LF component, it is worth recalling that Ponikowski and colleagues ${ }^{15}$ reported an increased risk of death only in patients with a reduced LF, whereas Szabo and colleagues ${ }^{14}$ reported a multivariate risk of death 2.5 times higher in patients with increased LF. These contrasting results reflect the poor applicability of spectral decomposition data obtained by automatic commercial Holter systems. ${ }^{1}$

The first prospective study on the prognostic implications of HRV in patients with cardiac failure (UK-HEART study) has been presented only in a preliminary form. ${ }^{17}$ In 433 ambulant outpatients with mild to moderate cardiac failure, 24 hour SDNN was significantly reduced in patients who died compared to those who survived. At multivariate analysis an arbitrary cut off value of $<100 \mathrm{~ms}$ emerged as a significant predictor of cardiac death but it failed to predict sudden death independently. This high cut off value was partially justified by the type of patients enrolled in the study (mean (SD) ejection fraction $42(17) \%$ ) but it remained far superior to that commonly used to stratify risk after myocardial infarction.

Although the results of this prospective study are not applicable to the usual cardiac failure population that often has more severe impairment of left ventricular function, they demonstrate that a simple HRV measure may contribute to better stratification of out-of-hospital patients with moderate cardiac failure when cardiac death is considered a primary end point. The lack of association between reduced HRV and sudden death once again confirms that depressed HRV predisposes more to an unfavourable progression of cardiac failure rather than to increased electrical instability of the myocardium.

\section{Conclusions}

It is noteworthy that these studies leave several doubts about the clinical usefulness of HRV assessment in stratifying patients with cardiac failure. Although further analyses with larger cohorts of patients and longer observation periods are necessary, available data indicate, in contrast to postmyocardial infarction patients, that HRV is not a powerful predictor of cardiac events. This may be explained by several considerations: (1) HRV is depressed in almost all patients with cardiac failure and it is known that parameters tending to very low levels have a reduced dispersion thus limiting their possible statistical power in survival regression models; (2) HRV indices reflect sympathetic-parasympathetic interactions, but expression of the disease at sinus node level may produce effects that are not related to the autonomic nervous system; (3) the presence of many confounding non-neural factors capable of a significant influence on HRV may weaken the common link, which has been proposed for normal subjects and postmyocardial infarction patients, between HRV and sympathetic or parasympathetic outflow to the heart, thus making the occurrence of arrhythmic death almost unpredictable.

F LOMBARDI

Cardiologia, Istituto Scienze Biomediche,

Ospedale S Paolo,

via A. di Rudiný 8

20142 Milano, Italy

email:lombfed@imiucca.csi.unimi.it

Cardiologia, Centro Medico di Montescano,

Fondazione S. Maugeriö,

IRCCS, Montescano, Pavia, Italy

1 Task Force of the European Society of Cardiology and the North American Society of Pacing and Electrophysiology. Heart rate variability. Standards of measurement, physiological interpretation, and clinical use. Circulation 1996;93:1043-65.

2 Guzzetti S, Cogliati C, Turiel M, et al. Sympathetic predominance followed by functional denervation in the progression of chronic heart failure. Eur Heart F 1995;16:1100-7.

3 Malliani A, Pagani M, Lombardi F, et al. Cardiovascular neural regulation explored in the frequency domain. Circulation 1991;84:482-92.

4 Mortara A, La Rovere MT, Signorini MG, et al. Can power spectral analysis of heart rate variability identify a high risk subgroup of congestive heart failure patients with excessive sympathetic activation? A pilot study before and after heart transplantation. Br Heart $\mathcal{F}$ 1994;71:422-30.

5 Lombardi $F$. The uncertain significance of reduced heart rate variability after myocardial infarction. Eur Heart f 1997;18:1204-6.

6 Lombardi F, Malliani A, Pagani M, et al. Heart rate variability and its sympatho-vagal modulation. Cardiovasc Res 1996;32:208-16.

7 Mortara A, Sleight P, Pinna GD, et al. Abnormal awake respiratory patterns are common in chronic heart failure and may prevent evaluation of autonomic tone by measures of heart rate variability. Circulation 1997;96:246-52

8 Binder T, Frey B, Porenta G, et al. Prognostic value of heart rate variability in patients awaiting cardiac transplantation. PACE 1992;15:2215-20.

9 Takase B, Kurita A, Noritake M, et al. Heart rate variability in patients with diabetes mellitus, ischemic heart disease, and congestive heart failure. $\mathcal{F}$ Electrocardiol 1992;25:79-88.

10 Woo MA, Stevenson WG, Moser DK, et al. Patterns of beat-to-beat heart rate variability in advanced heart failure. Am f Cardiol 1992;123:704-10.

11 Counihan PJ, Fei L, Bashir Y, et al. Assessment of heart rate variability in hypertrophic cardiomyopathy. Association with clinical and prognostic features. Circulation 1993;88:1682-90.

12 Brouwer J, van Veldhuisen DJ, Man in t'Veld AJ, et al, for the Dutch Ibopamine Multicenter Trial Study Group. Prognostic value of heart rate variability during long-term follow-up in patients with mild to moderate variability during long-term follow-up in patien
heart failure. $7 \mathrm{Am}$ Coll Cardiol 1996;28:1183-9.

13 Mortara A, Tavazzi L. Prognostic implications of autonomic nervous system analysis in chronic heart failure: role of heart rate variability and baroreflex sensitivity. Arch Gerontol Geriatr 1996;23:265-75.

14 Szabo BM, van Veldhuisen DJ, van der Veer N, et al. Prognostic value of heart rate variability in chronic congestive heart failure secondary to idiopathic or ischemic dilated cardiomyopathy. Am f Cardiol 1997;79:978-80.

15 Ponikowski P, Anker SD, Chua TP, et al. Depressed heart rate variability as an independent predictor of death in chronic congestive heart failure secondary to ischemic or idiopathic dilated cardiomyopathy. Am f Cardiol 1997;79:1645-50.

16 Jiang W, Hathaway WR, McNulty S, et al. Ability of heart rate variability to predict prognosis in patients with advanced congestive heart failure. $A m \mathcal{F}$ Cardiol 1997;80:808-11.

17 Nolan J, Andrews R, Brooksby P, et al. Relationship between heart rate variability and mode of death in chronic heart failure: results of the UK-HEART study [abstract]. Eur Heart f 1997;18:577(a). 